\title{
The Ultrastructure of the Gastrodermis and the Nutrition of the Gill Parasitic Atriaster heterodus Lebedev and Paruchin, 1969 (Platyhelminthes: Monogenea)
}

\author{
Cláudia P Santos/ ${ }^{+}$, Thais Souto-Padrón*, Reinalda M Lanfredi**
}

\begin{abstract}
Universidade Santa Úrsula, Instituto de Ciências Biológicas e Ambientais, Rua Fernando Ferrari 75, 22231-040
Rio de Janeiro, RJ, Brasil *Laboratório de Protozoologia I **Laboratório de Helmintologia, Instituto de Biofísica Carlos Chagas Filho, CCS, Bl. G, Universidade Federal do Rio de Janeiro, Ilha do Fundão, 21949-900 Rio de Janeiro, RJ, Brasil
\end{abstract}

The gastrodermis of Atriaster heterodus Lebedev \& Paruchin, 1969 (Polyopisthocotylea), a gill parasite from Diplodus argenteus (Valenciennes, 1830), is composed of " $U$ "-shape hematin cells and a connecting syncytium, both having cytoplasmic lamellae. These cells show outgrowths and bent folds which were seen to enclose lumen material. The trapped material was then subjected to endocytosis. The nature of ingested food material was comparatively analyzed by cytochemical and histochemical tests. Blood residues were detected in the gut but tests for mucins were negative. No intact erythrocytes were observed in the gut lumen.

Key words: Atriaster heterodus - Diplodus argenteus - Monogenea - fish - Brazil - nutrition

The functional morphology of the digestive system has been studied for different Monogenea (Smyth \& Halton 1983, Bogitsh 1993) and the feeding in polyopisthocotyleans and monopisthocotyleans is generally distinct with the former ingesting blood (Llewelyn 1954, Halton 1982) and the latter, mucus and epithelial cells. However, blood pigments were observed in a few monopisthocotyleans (Uspenskaya 1962, Kearn 1963, Fournier 1978, Buchmann et al. 1987) while Allen and Tinsley (1989) demonstrated blood and epithelial cells at the same time in the gut lumen of a polyopisthocotylean species.

Atriaster heterodus Lebedev \& Paruchin, 1969 (Polyopisthocotylea) was recently reported from Rio de Janeiro coast (Santos et al. 1996). Surface topography and ultrastructural aspects of the spermatogenesis of this species have been described by Santos et al. (1997). The ultrastructure of the gastrodermis and the nutrition of this species are described herein. This paper represents the first ultrastructural study of gut caeca and nutrition of a marine monogenean from Brazil.

Financial support by CNPq (Conselho Nacional de Pesquisa) and PRONEX (Programa de Núcleos de Excelência).

${ }^{+}$Corresponding author. Fax: +55-21-551.6446. E-mail: cpsantos@ax.apc.org

Received 19 November 1997

Accepted 8 January 1998

\section{MATERIALS AND METHODS}

Parasites - Sixty five specimens of A. heterodus were obtained from the gills of 25 Diplodus argenteus (Valenciennes, 1830) Sparidae, collected in Copacabana beach, Rio de Janeiro, Brazil.

Histochemistry - Monogeneans and gill filaments were fixed in 5\% buffered formalin or 70\% ethanol. Samples were embedded in paraffin and cut in $5 \mathrm{mM}$ sections which were deparaffinized with xylene and prior to staining, hydrated in decreasing concentrations of ethanol to water. Histochemical reactions are indicated with the references and color of positive reactions as follows. Neutral carbohydrate complexes: PAS (MacManus \& Mowry 1960) (+=various shades of purplishred); acidic carbohydrates: Alcian Blue (AB) and AB-PAS techniques (Mowry 1963) (+=blue); hemoglobin and derived pigments: Turnbull and Perls modified by Gomori (McManus \& Mowry 1960) (+=blue) and after Lilly and Fullmer (1976) were tested Perls (+=blue-green), Okajima ( $+=$ orange-red) and Dunn-Thompson $(+=$ emerald green). The picric alcohol solubility test (Llewelyn 1954) was tested for hematin (+=solve).

Ultrastructure - For ultrastructural studies, parasites were fixed for $2 \mathrm{hr}$ at $4^{\circ} \mathrm{C}$ in a solution containing $4 \%$ paraformaldehyde, $2.5 \%$ glutaraldehyde and $0.3 \mathrm{M}$ sucrose in $0.1 \mathrm{M}$ cacodylate buffer in sea water, $\mathrm{pH}$ 7.2. After being washed in the same solution, they were post-fixed for $1 \mathrm{hr}$ in dark conditions in a solution containing $1 \% \mathrm{OsO}_{4}$, $0.8 \%$ potassium ferricyanide, and $5 \mathrm{mM} \mathrm{CaCl}_{2}$ in 
$0.1 \mathrm{M}$ cacodylate buffer, dehydrated in acetone, and embedded in Epon. Ultrathin sections were picked up on uncoated 200-mesh copper grids, stained with uranyl acetate and lead citrate, and observed in a Zeiss 900 or Jeol JEM 100CX Electron Microscope. Some ultrathin sections were subjected to the picric acid/alcohol solubility test for hematin (Halton et al. 1968). Control sections were incubated in acid-free alcohol.

Cytochemistry - For basic protein detection, parasites were fixed as for ultrastructural studies, dehydrated in ethanol and incubated in a solution containing $2 \%$ phosphotungstic acid in absolute ethanol (E-PTA) for $2 \mathrm{hr}$ at room temperature (Bloom \& Aghajanian 1968). Specimens were rinsed in absolute ethanol, incubated for $10 \mathrm{~min}$ in propylene oxide and embedded in Epon to be observed with no counter stain. To test the presence of mucopolysacharides (Benhnke \& Zelander 1970), parasites were fixed for 1 to $18 \mathrm{hr}$ at room temperature in a solution containing $4 \%$ glutaraldehyde and $1 \%$ Alcian blue in sea water. Samples were washed twice for $10 \mathrm{~min}$ in sea water, and were post-fixed for $2 \mathrm{hr}$ at room temperature in dark conditions in a solution containing $1 \% \mathrm{OsO}_{4}$, $0.8 \%$ potassium ferricyanide and $5 \mathrm{mM} \mathrm{CaCl}_{2}$ in $0.1 \mathrm{M}$ cacodylate buffer $+3 \%$ sucrose. Parasites were, then, dehydrated in acetone and embedded in Epon. Ultrathin sections were picked up on uncoated 200 mesh copper grids, stained with uranyl acetate and lead citrate.

\section{RESULTS}

The digestive caeca of A. heterodus are highly branched and interspersed within vitellaria. Their folded gastrodermis consists of isolated hematin cells in a connecting syncytium.

Hematin cells appear elongated, "U"-shaped in longitudinal sections or round with closed borders in cross section (Figs 1-2). The basal nuclei have conspicuous heterochromatin. Mitochondria and numerous vesicles of irregular shape and varying electrondensity are located in the cytoplasm. The membrane in contact with the gut lumen forms numerous long lamellae enlarging the external cell surface (Figs 2-3). The hematin cells are located at irregular intervals along the gut. They are merged in the connecting syncytium composed of cells with scattered elongate nuclei, thin cytoplasm and few organelles. The syncytium also surrounds the hematin cells laterally and, in these areas, free lamellae, smaller than those of the hematin cells, can be observed on its surface (Fig. 3). Muscular fibers and parenchyma lie underneath the gastrodermis (Fig. 4).
Hematin cells and syncytium show outgrowths and bent folds which were seen to enclose lumen material with the help of the surface lamellae. The trapped material was then subjected to endocytosis (Fig. 4). Residual pigments remain inside of the vesicles in the external region of the cell. The way they are released from the cell was not observed. No free hematin cell was observed in the gut lumen.

The nature of hematin residues was confirmed by the histochemical test of picric acid in ethyl alcohol. The Turnbull method comparatively applied in the parasite and fish gill filaments confirmed the distribution of ferrous iron in hematin cells. The methods of Perls, Okajima and Dunn-Thompson were not conclusive. There was no reaction for mucin using the PAS and Alcian blue methods. Hematin cells stained with E-PTA displayed a strongly positive reaction in nuclear heterochromatin and in the small vesicles located at the external edge of the cell. Vesicles containing hematin residues were not labeled. A collection of membrane tubules and flattened sacs extends throughout the cytoplasm of the hematin cells.

\section{DISCUSSION}

The gastrodermis of A. heterodus is quite similar to the pattern described for other Polyopisthocotylea, with two cell types: hematin cell and connecting syncytium (Llewelyn 1954, Halton \& Jennings 1965, Halton et al. 1968, Tinsley 1973, Halton 1975).

Longitudinal sections showed that the irregular "U"-shaped hematin cells, are different from those previously reported for polyopisthocotyleans (Halton et al. 1968, Fournier 1978, Allen \& Tinsley 1989). The feeding process is continuous, with lamellae looping back onto the surface to capture and endocyte food particles, in a process similar to that described by Halton (1974), actually called micropinocytosis. Fournier (1978) also mentioned the endocytosis of gut content but did not mention the importance of the lamellae in this process.

The chemical nature of the pigments observed in the endocytosed vesicles was initially tested according to its solubility in picric acid in ethyl alcohol (Llewellyn 1954). Knowing that both formolic (formaldehyde) and hematin (hemoglobin) pigments are solved by picric acid and that formolic pigments do not react in the presence of Prussian blue (Perls), Turnbull blue, ferricyanide and ferrocyanide, contrary to what happen to hematin pigments (Lillie \& Fullmer 1976), these tests were applied in samples fixed either in $70 \%$ ethanol and buffered formalin. As in sample fixed in 70\% ethanol, where absence of formolic pigment is ex- 


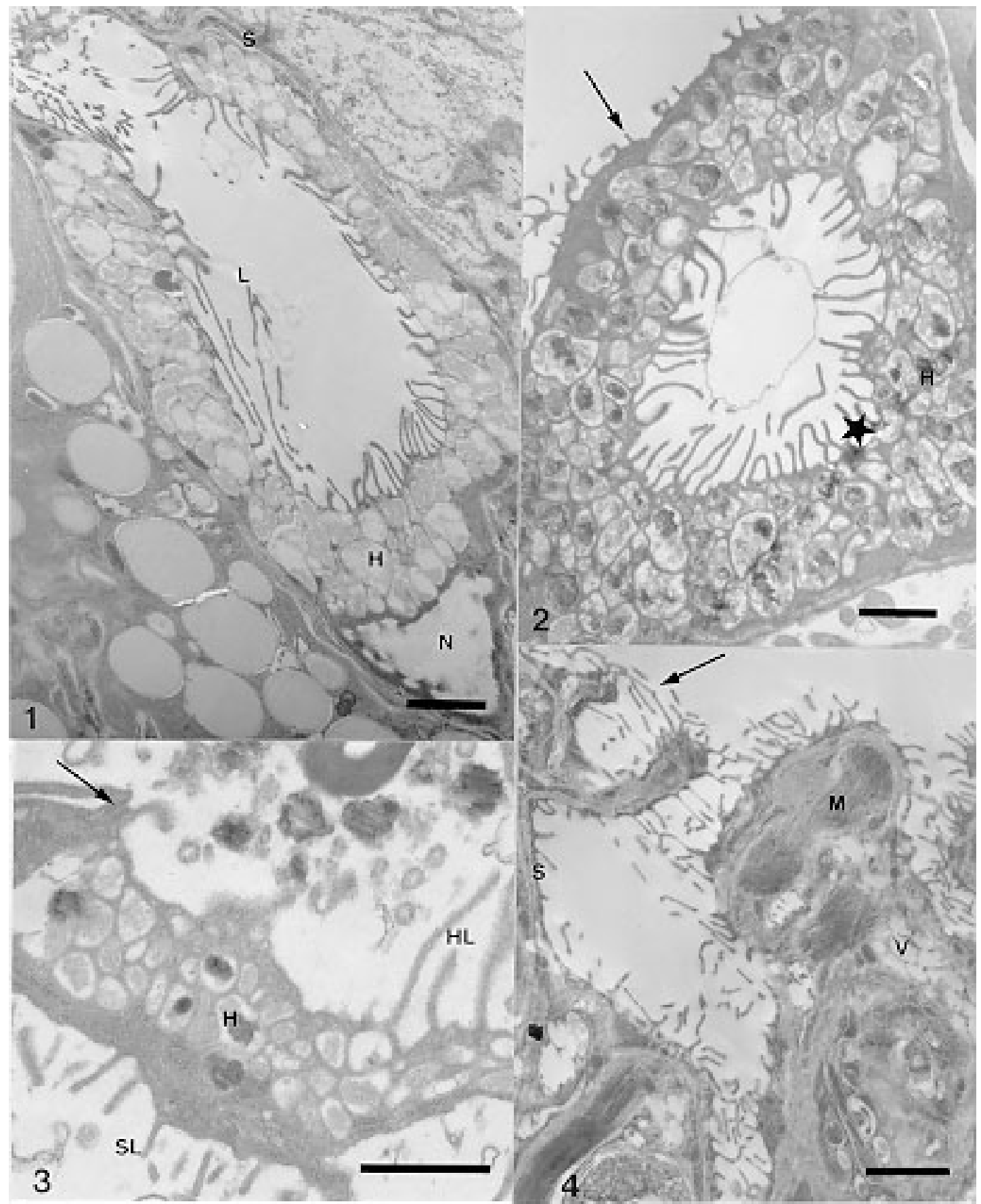

Gut lumen of Atriaster heterodus. Fig. 1: E-PTA cytochemistry in a "U"-shape hematin cell with basal nucleus (N), lamellae (L), connecting syncytium (S) and vesicles with hematin pigments (H). Fig. 2: cross section of hematin cell with lamellae (star) and vacuoles with hematin pigments $(\mathrm{H})$. Syncytium with small lamellae (arrow) are seen externally. Fig. 3: syncytium with small lamellae (SM) and junction (arrow) surrounding the hematin cell with vacuoles with hematin pigments $(\mathrm{H})$ and longer lamellae (HL). Fig. 4: E-PTA showing outgrowths of hematin cells with lamellae closing the borders (arrow). Muscular fibers (M), syncytium (S) and vesicles (V). Scale bars: Fig. $1=3 \mathrm{~mm}$; Figs $2-4=1 \mathrm{~mm}$. 


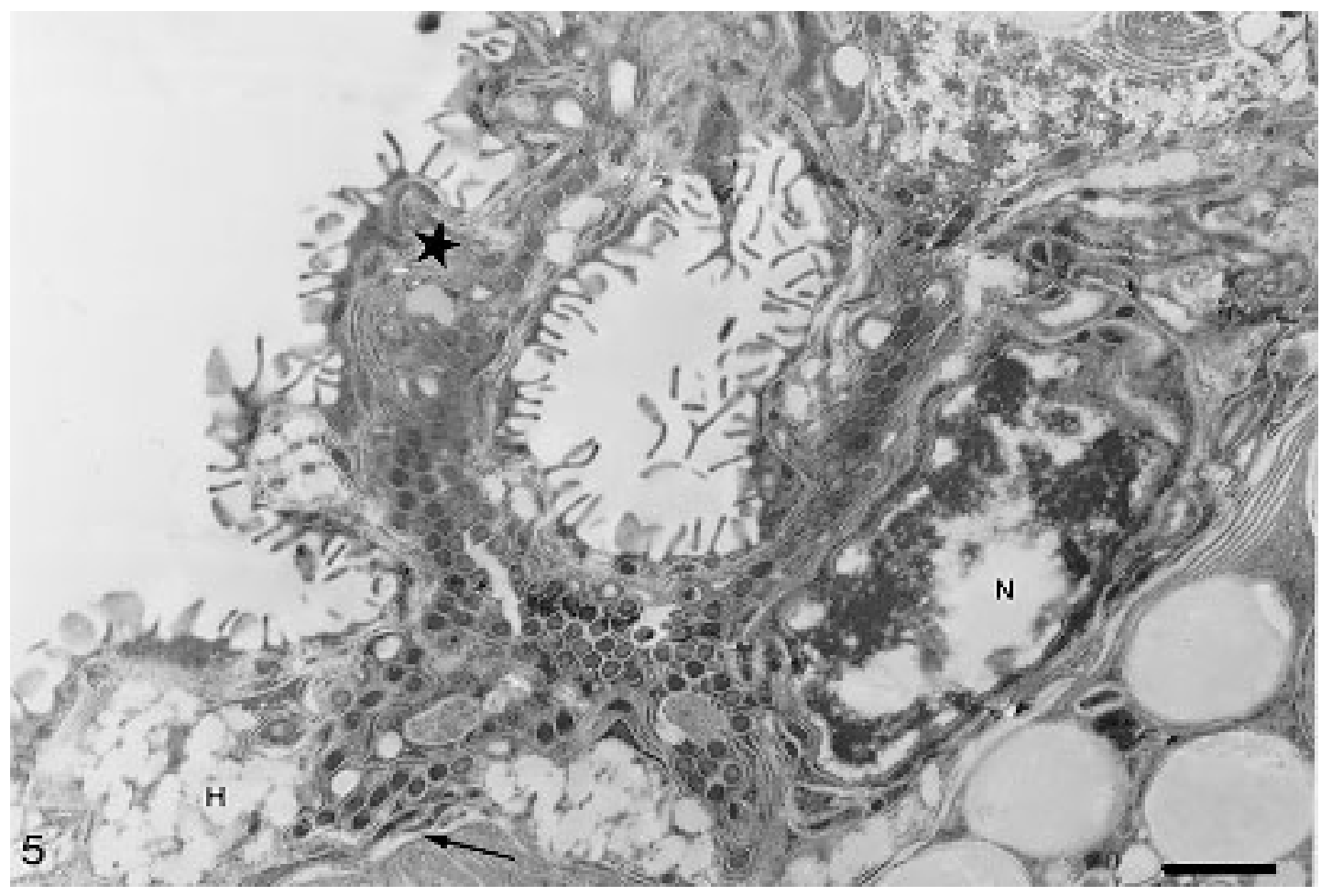

Fig. 5: E-PTA in gut lumen exhibiting an hematin cell with nucleus $(\mathrm{N})$, vacuoles with pigments of hematin not labeled $(\mathrm{H})$, system of membrane tubules and sacs in the cytoplasm (star) and a basal syncytium (arrow). Scale bar $=1 \mathrm{~mm}$

pected, the pigments were solubilized by the picric acid and also reacted for the Turnbull method, it was stated that the pigment was in fact hematin. The histochemistry and cytochemistry tests, negative for mucins and positive for iron residues, confirmed the chemical nature as hematin pigments, indicating hematophagous food habit for this parasite. Host hemoglobin in vacuoles strongly suggest that at least intracellular digestion occurs in these cells.

No intact erythrocytes were observed in the gut. This can be explained by fish handling. The monogeneans were collected some hours after fish capture and according to Llewellyn (1954) erythrocytes can only be observed in their gut when fish are examined in less than one hour after capture, because a rapid haemolysis followed by phagocytosis normally occur. However, it cannot be excluded that erythrocytes are lysed during the feeding process.

The structure of the syncytium cells with or without lamellae was previously reported (Halton et al. 1968, Tinsley 1973). Nevertheless, in both cases these monogeneans that feed on blood present a syncytium with few spread organelles, function- ing mainly as a support for the hematin cells, on the contrary to those that present a mixed food habit like Polystomoides sp., where the syncytial cytoplasm has numerous organelles (Allen \& Tinsley 1989). The reduced syncytial cytoplasm of $A$. heterodus with few organelles presupposes a support function but their role in the feeding process is still doubtful.

This is the first study on the ultrastructure of the gastrodermis of A. heterodus with detection of its haematophagous food habit.

\section{ACKNOWLEDGEMENTS}

To Dr Kurt Buchmann (Royal Veterinary and Agriculture University, Denmark) and Dr Walter A Boeger (Universidade Federal do Paraná, Brazil) who kindly commented on the manuscript.

\section{REFERENCES}

Allen KM, Tinsley RC 1989. The diet and gastrodermal ultrastructure of polystomatid monogeneans infecting chelonians. Parasitology 98: 265-273.

Benhnke O, Zelander T 1970. Preservation of intercellular substances by the cationic dye Alcian blue in preparative procedures for electron microscopy. $J$ Ultrastruct Res 31: 424. 
Bloom FE, Aghajanian GK 1968. Fine structure and cytochemical analysis of the staining of synaptic junctions with phosphotungstic acid. J Ultrastruct Res 22: 361-375.

Bogitsh BJ 1993. A comparative review of the flatworm gut with emphasis on the Rhabdcoela and Neodermata. Trans Amer Microsc Soc 112: 1-9.

Buchmann K, Koie M, Prento P 1987. The nutrition of the gill parasitic monogenean Pseudactylogyrus anguillae. Parasitol Res 73: 532-537.

Fournier A 1978. Euzetrema knoepffleri: evidence for a synchronous cycle of gastrodermal activity and an "apocrine-like" release of the residues of digestion. Parasitology 77: 19-26.

Halton DW 1974. Hemoglobin absorption in the gut of a monogenetic trematode, Diclidophora merlangi. J Parasitology 60: 59-66.

Halton DW 1975. Intracellular digestion and cellular defecation in a monogenean Diclidophora merlangi. Parasitol 70: 331-340.

Halton DW 1982. X-ray microanalysis of pigment granules in the gut of $D$. merlangi (Monogenoidea). Zeitsch Parasit 68: 113-115.

Halton DW, Jennings, JB 1965. Observations on the nutrition of monogenetic trematodes. Biol Bull 129: 257-272.

Halton DW, Dermott E, Morris GP 1968. Electron microscope studies of Diclidophora merlangi (Monogenea: Polyopisthocotylea). I. Ultrastructure of the cecal epithelium. J Parasitol 54: 909-916.

Kearn GC 1963. Feeding in some monogenean skin parasites: Entobdella solea on Solea solea and Acanthocotyle sp. on Raia clavata. J Mar Biol Ass United Kingdom 43: 749-766.
Lillie RD, Fullmer HM 1976. Histopathologic Technique and Practical Histochemistry, 4th ed., MacGraw-Hill Book Company, New York, 850 pp.

Llewellyn J 1954. Observations on the food and gut pigment of the Polyopisthocotylea (Trematoda: Monogenea). Parasitology 44: 428-437.

McManus JFA, Mowry RW 1960. Staining Methods. Histologic and Histochemical, Paul B, Hoeber, Inc. Medical Division of Harper \& Brothers, 423 pp.

Mowry RD 1963. The special value of methods that color both acidic and vicinal hydroxyl groups in the histhochemical study of mucins with revised directions for the coloidal iron stain, the use of Alcian blue G8X and their combinations with the periodic acidSchiff reaction. Ann NY Acad Sci 106: 402-423.

Santos CP, Lanfredi RM, Souto-Padrón T 1997. Ultrastructure of spermatogenesis of Atriaster heterodus (Platyhelminthes, Monogenea, Polyopisthocotylea). J Parasitol 83: 1007-1014.

Santos CP, Souto-Padrón T, Lanfredi RM 1996. Atriaster heterodus Lebedev \& Paruchin, 1969 and Polylabris tubicirrus (Paperna \& Kohn, 1964) (Monogenea) from Diplodus argenteus (Val., 1830) (Teleostei: Sparidae) from Brazil. J Helm Soc Washington 63: 181-187.

Smyth J, Halton DW 1983. The Physiology of Trematodes, Cambridge University Press, Cambridge, 446 pp.

Tinsley RC 1973. Ultrastructural studies on form and function of the gastrodermis of Protopolystoma xenopi (Monogenoidea: Polyopisthocotylea). Biol Bull 144: 541-555.

Uspenskaya AV 1962. Nutrition of monogenetic trematodes. Dok Akad Nauk USSSR 142: 1212-1215. 
282 Gastrodermis and Nutrition of $A$. heterodus - Cláudia P Santos et al. 